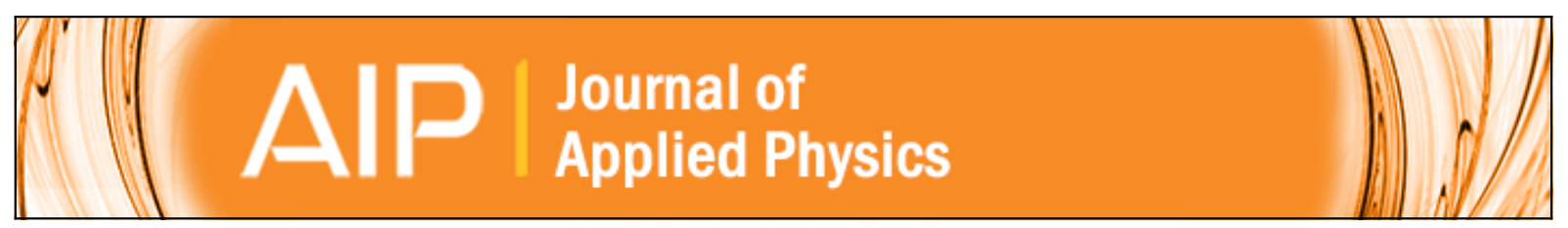

\title{
Optical anisotropy in bismuth titanate: An experimental and theoretical study
}

Amritendu Roy, Rajendra Prasad, Sushil Auluck, and Ashish Garg

Citation: Journal of Applied Physics 115, 133509 (2014); doi: 10.1063/1.4870460

View online: http://dx.doi.org/10.1063/1.4870460

View Table of Contents: http://scitation.aip.org/content/aip/journal/jap/115/13?ver=pdfcov

Published by the AIP Publishing

\section{Articles you may be interested in}

Raman spectra and dielectric function of BiCrO3: Experimental and first-principles studies

J. Appl. Phys. 110, 073501 (2011); 10.1063/1.3642985

Dielectric and ferroelectric properties of c -axis oriented strontium bismuth tantalate thin films applied transverse electric fields

J. Appl. Phys. 99, 124106 (2006); 10.1063/1.2205351

Epitaxial BiFeO 3 thin films on $\mathrm{Si}$

Appl. Phys. Lett. 85, 2574 (2004); 10.1063/1.1799234

Structural and electrical anisotropy of (001)-, (116)-, and (103)-oriented epitaxial SrBi 2 Ta 209 thin films on SrTiO 3 substrates grown by pulsed laser deposition

J. Appl. Phys. 88, 6658 (2000); 10.1063/1.1321776

Epitaxial growth of $\mathrm{SrBi} 2 \mathrm{Nb} 2 \mathrm{O} 9$ on (110) $\mathrm{SrTiO} 3$ and the establishment of a lower bound on the spontaneous polarization of $\mathrm{SrBi} 2 \mathrm{Nb} 2 \mathrm{O} 9$

Appl. Phys. Lett. 77, 3090 (2000); 10.1063/1.1322055

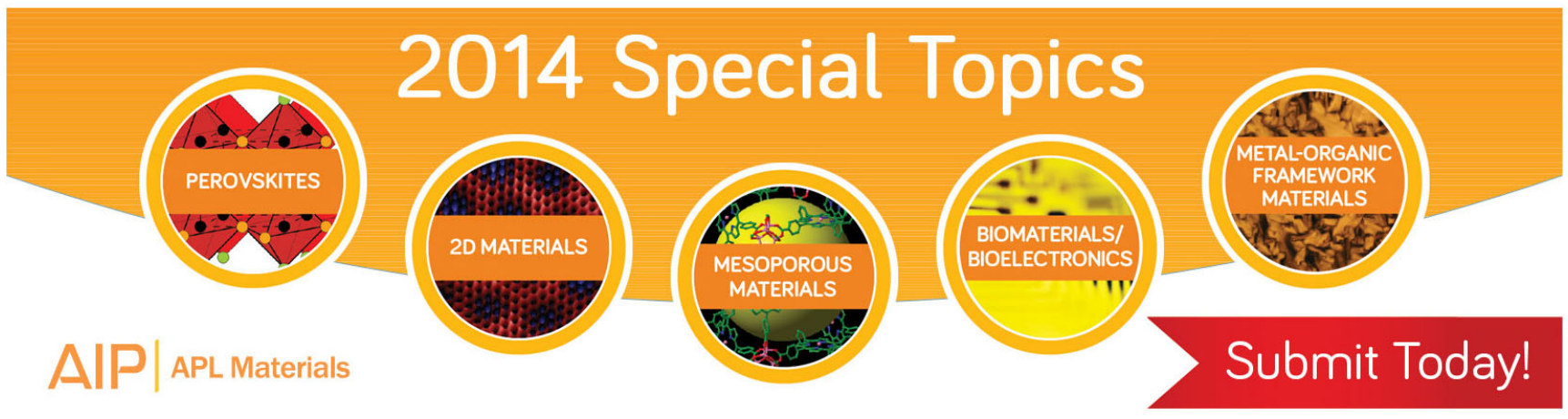




\title{
Optical anisotropy in bismuth titanate: An experimental and theoretical study
}

\author{
Amritendu Roy, ${ }^{1, a)}$ Rajendra Prasad, ${ }^{2}$ Sushil Auluck, ${ }^{3}$ and Ashish Garg ${ }^{1}$ \\ ${ }^{1}$ Department of Materials Science and Engineering, Indian Institute of Technology Kanpur, Kanpur-208016, \\ India \\ ${ }^{2}$ Department of Physics, Indian Institute of Technology Kanpur, Kanpur-208016, India \\ ${ }^{3}$ CSIR-National Physical Laboratory, K.S. Krishnan Marg., New Delhi, India
}

(Received 2 January 2014; accepted 24 March 2014; published online 3 April 2014)

\begin{abstract}
We report experimental and theoretical investigation of anisotropy in optical properties and their origin in the ferroelectric and paraelectric phases of bismuth titanate. Room temperature ellipsometric measurements performed on pulsed laser deposited bismuth titanate thin films of different orientations show anisotropy in the dielectric and optical constants. Subsequent first-principles calculations performed on the ground state structures of ferroelectric and high temperature paraelectric phases of bismuth titanate show that the material demonstrates anisotropic optical behavior in both ferroelectric and paraelectric phases. We further show that $\mathrm{O} 2 \mathrm{p}$ to $\mathrm{Ti} 3 \mathrm{~d}$ transition is the primary origin of optical property of the material while optical anisotropy results from the asymmetrically oriented $\mathrm{Ti}-\mathrm{O}$ bonds in $\mathrm{TiO}_{6}$ octahedra in the unit cell. (C) 2014 AIP Publishing LLC. [http://dx.doi.org/10.1063/1.4870460]
\end{abstract}

\section{INTRODUCTION}

Ferroelectric bismuth titanate $\left(\mathrm{Bi}_{4} \mathrm{Ti}_{3} \mathrm{O}_{12}\right.$ or $\left.\mathrm{BiT}\right)$ and related materials enjoy profound research interests from the perspective of their potential applications in ferroelectric random access memory (FeRAM), piezoelectric and electro-optical devices owing to their large remnant polarization, as well as environment friendly nature. ${ }^{1}$ Improved fatigue endurance upon lanthanide doping and lower processing temperature, in addition, make these materials as suitable alternatives to lead based ferroelectrics in the application domain where integration on standard silicon substrates has become the topic of research of device designing for improved performance. ${ }^{2,3}$ Dielectric, optical, and optoelectronic properties of BiT are of further interest in the area of optical memories since its band gap $\left(\mathrm{E}_{\mathrm{g}} \sim 3.6 \mathrm{eV}\right),{ }^{4}$ similar to most ferroelectric perovskite oxides, lies in the visible spectrum region. As a result, dielectric ${ }^{5-13}$ and optical properties ${ }^{13-19}$ of BiT have been extensively investigated for decades. In addition, there has been a renewed interest in the optical properties of ferroelectric BiT as noncentrosymmetric crystal structure of room temperature BiT could trigger asymmetric electron excitation, relaxation, and scattering leading to photovoltaic effect. ${ }^{20,21}$

Ferroelectric BiT with layered perovskite structure has a complex crystal structure (with monoclinic Blal symmetry $)^{22}$ consisting of alternate stacking of fluorite $\left(\mathrm{Bi}_{2} \mathrm{O}_{2}\right)^{2+}$ and perovskite-like $\left(\mathrm{Bi}_{2} \mathrm{Ti}_{3} \mathrm{O}_{7}\right)^{2-}$ layers arranged along the crystallographic $c$-axis. Room temperature lattice parameters are: $a=5.450(1) \AA, b=5.4059(6) \AA$, and $c=32.832(3) \AA .{ }^{4}$ At Curie temperature $\left(\mathrm{T}_{\mathrm{c}}=948 \mathrm{~K}\right)$, ferroelectric BiT transforms into a paraelectric phase with centrosymmetric tetragonal $(I 4 / \mathrm{mmm})$ structure having lattice parameters,

\footnotetext{
a) Author to whom correspondence should be addressed. Email address: tendur@gmail.com. Tel.: +91-9451018914; Fax: +91-512-2597505. Present address: School of Minerals, Metallurgical \& Materials Engineering, Indian Institute of Technology, Bhubaneswar, India.
}

$a=b=3.8524 \AA$ and $c=33.197 \AA{ }^{23}$ Noncentrosymmetry of the room temperature structure along with its complexity further renders anisotropic physical properties in the material, namely dielectric constant $\left(\varepsilon_{a}=\varepsilon_{b}=153 \pm 5\right.$ and $\varepsilon_{c}=$ $118 \pm 5$ at $100 \mathrm{kHz}),{ }^{6}$ ferroelectric polarization, ${ }^{15,24}$ and

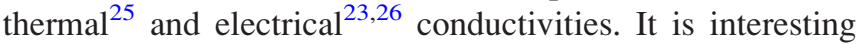
to note that anisotropy in different physical properties have different origins. For example, directional dependence of thermal properties is attributed to the density difference between the perovskite and the fluorite type $\left(\mathrm{Bi}_{2} \mathrm{O}_{2}\right)$ layers in the unit cell ${ }^{25}$ while anisotropy in the electrical properties arises due to differences in the oxygen vacancy concentrations in the perovskite and $\mathrm{Bi}_{2} \mathrm{O}_{2}$ oxide layer of the structure. $^{23,26}$ In contrast, anisotropy in the ferroelectric polarization is attributed to non-zero displacement of Ti ions along $a$-axis. ${ }^{24}$

From the perspective of optical devices utilizing oriented thin films of BiT, it is vital to experimentally and theoretically explore the optical anisotropy if any and then to understand its origin. Here, first-principles density functional calculations would be useful since such study could shed a detailed insight into the origin of optical property, as shown previously for a number of oxides. ${ }^{20,27-29}$ Though considerable attention has been paid toward the experimental studies of optical and dielectric properties of BiT, ${ }^{15,17,19}$ very few reports provide a microscopic understanding of the optical properties using first-principles calculations. Among the few reports, Cai et al. ${ }^{30}$ based on the bond orbital theory concluded that the large nonlinear refractive index of ferroelectric $\mathrm{BiT}$ is due to virtual electronic excitation from the filled valence band (VB) to the empty cationic $d$-orbital at short equilibrium bond lengths. Recently, optical properties of ferroelectric bismuth titanate have been calculated using TBmBJ functional using a smaller cell with space group $P C$ (Ref. 27) where close proximity between the experimental and calculated band gap was found; however, orientation dependence of optical behavior was not discussed. 
In this manuscript, we show the results of ellipsometric measurements on epitaxial bismuth titanate thin films of two different orientations grown on (100) and (110) oriented strontium titanate (STO) substrates using pulsed laser deposition. Optical constants determined by fitting the ellipsometric data demonstrate direction dependence pointing towards optical anisotropy in the material. Further, we calculated the optical properties in ferroelectric and paraelectric phases of bismuth titanate using first-principles density functional theory based calculations using local density approximation (LDA) and generalized gradient approximation (GGA) methods as well as full-potential based linear augmented Plane wave (LAPW) method using GGA technique. The calculations show orientation dependence of the dielectric function not only in the ferroelectric (Blal) phase, but also in the paraelectric $(14 / \mathrm{mmm})$ phase of BiT. We also show that the optical property in both the phases originates primarily from $\mathrm{O} 2 \mathrm{p}$ to $\mathrm{Ti} 3 \mathrm{~d}$ transition while observed anisotropy in the optical constants is attributed to the asymmetrically oriented $\mathrm{Ti}-\mathrm{O}$ bonds in $\mathrm{TiO}_{6}$ octahedra in the unit cell.

\section{EXPERIMENTAL CALCULATION AND DETAILS}

Thin films of BiT were grown using pulsed laser deposition technique on strontium titanate substrates of two different orientations viz. (100) and (110) from a stoichiometric target of BiT. The film growth was carried out using an excimer laser of wavelength $248 \mathrm{~nm} \quad(\mathrm{KrF})$ in an oxygen environment $\left(\mathrm{pO}_{2} \sim 0.40\right.$ mbar) at a substrate temperature of $750{ }^{\circ} \mathrm{C}$ using a laser fluence of $2.3 \mathrm{~J} / \mathrm{cm}^{2}$ and a repetition rate of $5 \mathrm{~Hz}$. Prior to the deposition, a base pressure of $2 \times 10^{-6}$ mbar was achieved. The films were annealed at the deposition temperature in an oxygen environment with $\mathrm{pO}_{2} \sim 0.67 \mathrm{mbar}$ for $30 \mathrm{~min}$ and then cooled to room temperature at the deposition pressure. $\mathrm{X}$-ray diffraction of the films was performed using a high resolution Philips X'Pert PRO MRD thin film diffractometer using $\mathrm{CuK} \alpha$ radiation over $2 \theta$ range of $10^{\circ}$ to $80^{\circ}$. Ellipsometric measurements on the two differently oriented films were performed to appreciate the effect of film's orientation on the optical property of the material. Ellipsometric measurements were carried out using HORIBA JOBIN-YVON spectroscopic ellipsometer (SE) over the energy range of $0.8-2.5 \mathrm{eV}$ with an incidence angle of $70^{\circ}$. In the present work, we have used a three layer model in which complex dielectric function and other optical properties were determined by simulating the experimental data using Tauc-Lorentz (TL) model. ${ }^{31}$ Separate ellipsometric measurements were performed on the bare substrates in order to separate out their contributions.

In order to substantiate the experimental observations and to identify the origin of optical response vis-à-vis optical anisotropy in bismuth titanate, we performed first-principles density functional calculations for the electronic structures and optical properties in the ferroelectric Blal and paraelectric $14 / \mathrm{mmm}$ phases. We carried out bulk calculations considering unit cells with 76 and 38 atoms for Blal and $I 4 / \mathrm{mmm}$ structures of BiT, respectively. We started our calculations with the optimized lattice parameters and ionic positions of Blal and $14 / \mathrm{mmm}$ phases of bismuth titanate calculated using GGA and LDA. ${ }^{24}$ In the above calculations, we, using the experimental structural parameters, relaxed the cell volume and ionic positions systematically in such a way that the forces on ions are less than the specified limit, and the total pressure on the system is close to zero. In the whole process, we restricted the symmetry of the system to be unaltered. ${ }^{24}$ The entire calculation was carried out in the framework of first-principles density functional theory. ${ }^{32}$ Vienna ab-initio simulation package (VASP) ${ }^{33,34}$ was used with projector augmented wave method (PAW). ${ }^{35}$ The Kohn-Sham equation $^{36,37}$ was solved using the exchange correlation function of Perdew and Wang ${ }^{38,39}$ for GGA and of Ceperley-Alder ${ }^{40}$ for LDA schemes. We included five valence electrons for $\mathrm{Bi}$ $\left(6 \mathrm{~s}^{2} 6 \mathrm{p}^{3}\right), 4$ for $\mathrm{Ti}\left(3 \mathrm{~d}^{3} 4 \mathrm{~s}^{1}\right)$, and 6 for $\mathrm{O}\left(2 \mathrm{~s}^{2} 2 \mathrm{p}^{4}\right)$. A plane wave energy cut-off of $400 \mathrm{eV}$ yielded good convergence of the total energy. Conjugate gradient algorithm ${ }^{41}$ was used for the structural optimization. All the calculations were performed at $0 \mathrm{~K}$. For electronic structure and optical property calculation, Monkhorst-Pack ${ }^{42} 8 \times 8 \times 8$ mesh was used, which proved good in our previous work on the same system. ${ }^{24}$ In order to substantiate our results using pseudopotential based approach, we repeated our entire calculations by full potential-linear augmented plane wave (FP-LAPW) method with GGA using WIEN2k. Such a comparison would be first of its kind for a complex system such as bismuth titanate.

\section{RESULTS AND DISCUSSION}

\section{A. Optical characterization using ellipsometry}

Fig. 1(a) plots XRD spectra of single phase BiT thin films (thickness $\sim 700-750 \mathrm{~nm}$ ) deposited on two differently oriented STO substrates. The bottom panel depicts (001)-oriented BiT with presence of (001) peaks. The top panel shows the film with (118)-orientation with major peak being (2 2 16) peak ((118) reflection is not seen due to systematic absences). A weaker (117) peak is also observed which is

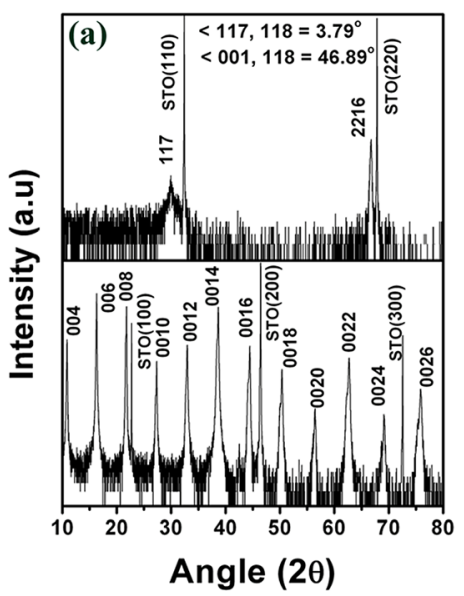

(b)
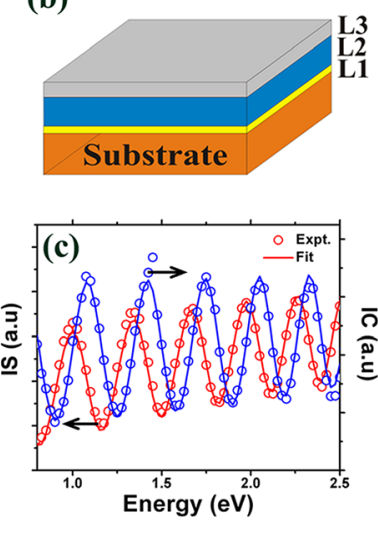

FIG. 1. (a) XRD of BiT thin films with orientations (001) (lower panel) and (118) (upper panel) deposited on $\mathrm{SrTiO}_{3}$ single crystalline substrates with orientations (100) and (110), respectively. (b) Three layer model used for simulating the experimental ellipsometry data. Above the substrate, L1 represents an interface between the substrate and BiT film, L2 shows BiT thin film, and L3 indicates top surface of the film with some roughness. (c) Simulation of ellipsometry data of (001) oriented BiT film using Tauc-Lorez model, shown the validity of the present model. 
quite close to the calculated position of (118) peak. On the other hand, (001) and (118) are $\sim 47^{\circ}$ apart from each other. While a difference in the lattice parameters of BiT and $\mathrm{SrTiO}_{3}$ suggests that the film should possess a tensile misfit strain of $\sim 2 \%$, relatively higher film thickness of $\sim 750 \mathrm{~nm}$ and post growth annealing are likely to result in a strain relaxed film to its bulk dimensions. Subsequently, we performed ellipsometric measurements on these two differently oriented films to study the effect of orientation on their linear optical responses. Fig. 1(b) shows the three layer model used for fitting the ellipsometric data using TL model. ${ }^{31}$ Fig. 1(c) shows the plots of experimental and fitted data for (001) oriented BiT film, suggesting validity of the model used in the present study. Calculated film thickness is consistent with the profilometer data $(\sim 700-750 \mathrm{~nm})$. Obtained band gap is also consistent with previous reports. ${ }^{43}$

Subsequently, real and imaginary components of dielectric spectra obtained from the fitting were plotted for the two films as shown in Fig. 2(a). Since both the films have
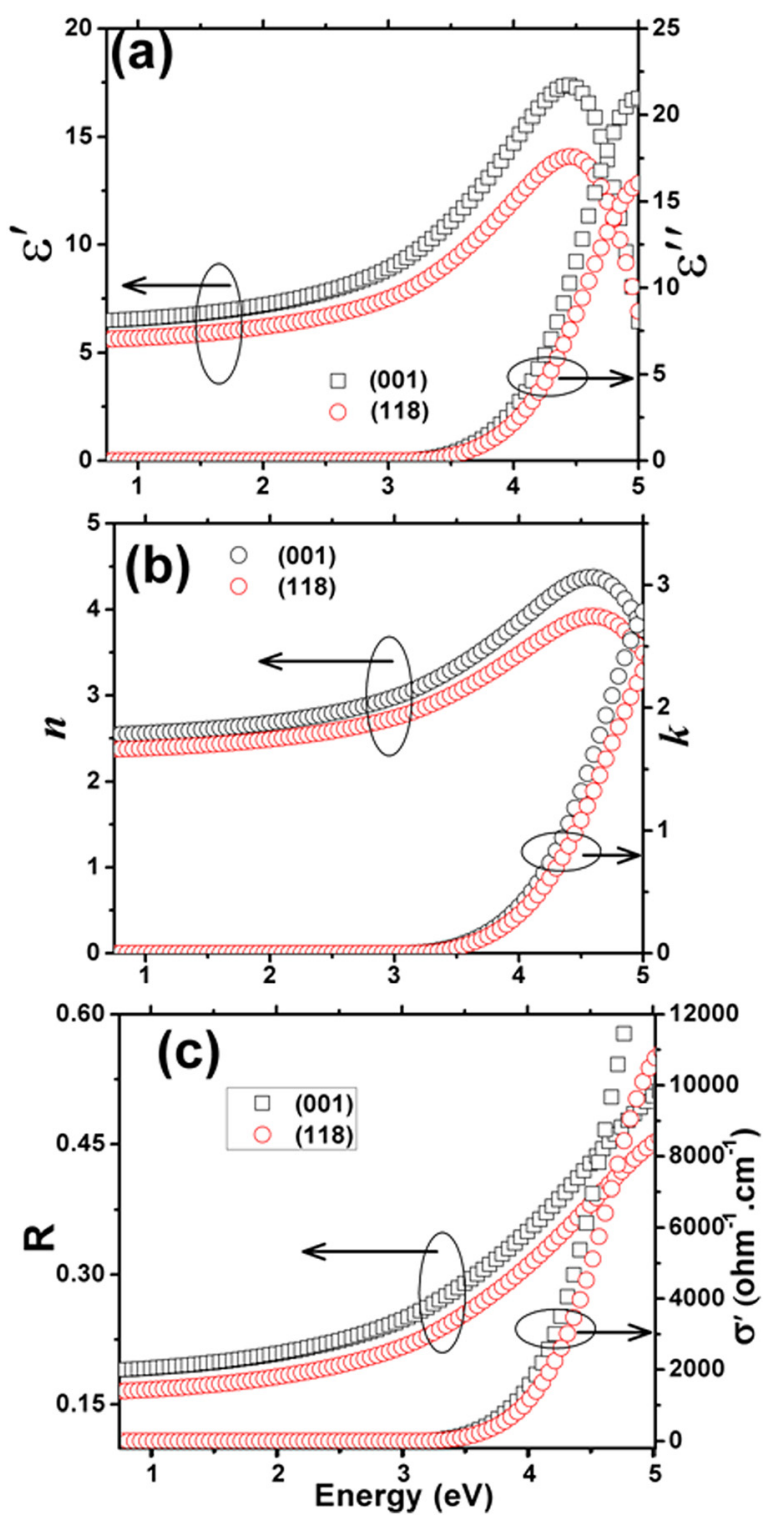

FIG. 2. Optical constants of BiT thin films with (001) and (118) orientations obtained from ellipsometric measurements.

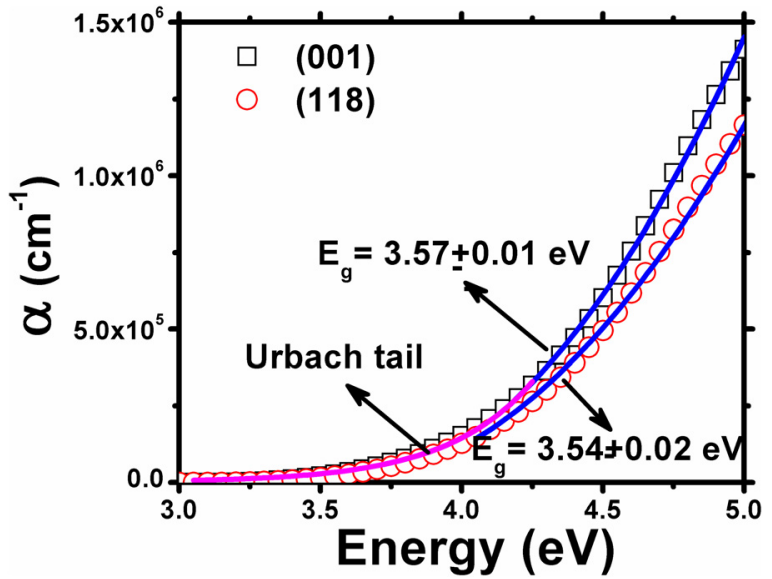

FIG. 3. Absorption coefficient as a function of incident photon energy of BiT thin films with (001) and (118) orientations obtained from ellipsometric measurements.

approximately identical thicknesses, optical response can also expected to be similar provided the material was isotropic. In contrast, dielectric spectra of the two films, as shown in Fig. 2(a), demonstrate different intensities translating into different optical constants for the two films. Fig. 3 plots absorption coefficients $(\alpha)$ as a function of energy for the two films. For semiconductors, absorption coefficient follows $\mathrm{E}^{1 / 2}$ and $\mathrm{E}^{2}$ relationships for direct and indirect gap semiconductors, respectively. We find that the absorption spectra beyond the band gap of both the films follow Eq. (1) nicely as shown below

$$
\alpha=\alpha_{0}\left(\frac{E-E_{g}}{E_{g}}\right)^{2}
$$

where $E_{g}$ represents the band gap. This substantiates that $\mathrm{BiT}$ is an indirect band gap semiconductor, in agreement with our previous work ${ }^{24}$ and several other experiments ${ }^{19,43}$ and first-principles calculations. ${ }^{27}$ Upon fitting our absorption data using Eq. (1), we obtained energy band gap of the order of $\mathrm{E}_{\mathrm{g}}=3.57 \pm 0.01 \mathrm{eV}$ and $\mathrm{E}_{\mathrm{g}}=3.54 \pm 0.02 \mathrm{eV}$ for (001) and (118) oriented films, respectively, which are consistent with previously reported data. ${ }^{43}$ However, bandgap of polycrystalline thin films of $\mathrm{BiT}$ is $\mathrm{E}_{\mathrm{g}} \sim 3.41 \mathrm{eV}{ }^{44}$ Below the band gap, our samples manifest phonon assisted Urbach absorption, which can be conveniently modelled by

$$
\alpha=\alpha_{g}\left(\frac{E-E_{g}}{E_{U}}\right),
$$

where $\mathrm{E}_{\mathrm{U}}$ indicates the Urbach absorption energy. Use of Eq. (2) in the low absorption Urbach region yield $\mathrm{E}_{\mathrm{g}}=3.64 \pm 0.02 \mathrm{eV}$ for (001) oriented film. Subsequent calculations of optical constants such as refractive index $(n)$, extinction coefficient $(k)$, and reflectivity $(R)$ for the two differently oriented films are presented in Figs. 2(b) and 2(c). These figures show different optical behaviors for the above two films again pointing towards optical anisotropy in BiT. For instance, refractive indices for the two films when extrapolated to zero frequencies are 2.44 and 2.27, respectively, for (001) and (118) orientations. These values are consistent with 
previous experiments ${ }^{43}$ as well as previous birefringence data on single crystals of bismuth titanate and substantiate our results showing anisotropy in refractive indices along different crystallorgraphic directions at $25^{\circ} \mathrm{C} .{ }^{15}$ Reflectivity and optical conductivity too demonstrate different magnitudes when plotted as a function of incident energy.

In order to develop an in-depth understanding of the observed optical response in ferroelectric phase of BiT, we further performed first-principles calculations on the ground state structure of BiT in its ferroelectric state. In addition, we also carried out first-principles calculations for optical properties on the high temperature paraelectric phase of BiT.

\section{B. First-principles calculations and the origin of optical anisotropy}

Linear response of a material to an external electromagnetic field with a small wave vector could be determined by the complex dielectric function, $\varepsilon(\omega)$. Since we are interested in the optical response of the material, the frequencies of our interests lie in the optical frequency range, viz., $\sim 10^{13}-10^{16} \mathrm{~Hz}$. Time-dependent perturbations of the ground-state electronic states can be used to describe the interaction of photon (associated with the electromagnetic field) and the electrons of the material. The resulting optical transitions between occupied and unoccupied electronic states which result from the electric field of the photon give rise to the observed optical spectra. Optical spectra can therefore be expressed in terms of the joint density of states between the valence and conduction bands (CBs). Eigen vectors obtained from the solution of the Schrödinger equation can provide the valence and conduction bands, which are further used to determine the momentum matrix elements and subsequently the complex dielectric function. The imaginary part of the complex dielectric function $\left(\varepsilon^{\prime \prime}(\omega)\right)$ can thus be written as

$$
\begin{aligned}
\varepsilon^{\prime \prime}(\omega)= & \left(\frac{V e^{2}}{2 \pi \hbar m^{2} \omega^{2}}\right) \int d^{3} k \sum_{n n^{\prime}}\left|\left\langle\mathrm{k} n|\mathrm{p}| \mathrm{k} n^{\prime}\right\rangle\right|^{2} f(\mathrm{k} n) \\
& \times\left(1-f\left(\mathrm{k} n^{\prime}\right) \delta\left(\mathrm{E}_{\mathrm{K} n}-\mathrm{E}_{\mathrm{K} n^{\prime}}-\hbar \omega\right),\right.
\end{aligned}
$$

where $\mathrm{p}$ is the momentum operator, $|\mathrm{k} n\rangle$ is crystal wave function, $f(\mathrm{k} n)$ is Fermi function, and $\hbar \omega$ is the incident photon energy. The real part of the dielectric function $\varepsilon^{\prime}(\omega)$ can be determined from $\varepsilon^{\prime \prime}(\omega)$ using Kramers-Kronig relation ${ }^{45}$

$$
\varepsilon^{\prime}(\omega)=1+\frac{2}{\pi} \int_{0}^{\infty} \frac{\varepsilon^{\prime \prime}\left(\omega^{\prime}\right) \omega^{\prime} d \omega^{\prime}}{\omega^{\prime 2}-\omega^{2}} .
$$

In crystalline materials, dielectric function is expressed by a second rank tensor where the elements are dependent on owing to crystal symmetry. In the ferroelectric Blal symmetry of BiT, the real and imaginary parts of the dielectric function tensor would have three unequal leading diagonal elements, $\varepsilon_{\mathrm{xx}}, \varepsilon_{\mathrm{yy}}$, and $\varepsilon_{\mathrm{zz}}$ along with off-diagonal term, $\varepsilon_{\mathrm{zx}}$, due to monoclinic symmetry. The off-diagonal elements do not contribute to optical anisotropy but give rise to optical rotation or optical activity. ${ }^{46,47}$ Since the monoclinic distortion in the present system is very small $\left(\beta=90.08^{\circ}\right){ }^{24}$ the off-diagonal element $\varepsilon_{\mathrm{zx}}$ when determined as a function of incident photon energy is very small in comparison to the diagonal $\left(\varepsilon_{\mathrm{xx}}, \varepsilon_{\mathrm{yy}}\right.$, and $\left.\varepsilon_{\mathrm{zz}}\right)$ elements. We, therefore, ignore the off-diagonal elements and present only the diagonal elements. Paraelectric BiT, on the other hand, has tetragonal $(I 4 / \mathrm{mmm})$ symmetry; and therefore, there would be two components of real and imaginary parts of dielectric function

$$
\left.\begin{array}{rl}
\varepsilon_{\perp} & =\frac{\varepsilon_{x x}+\varepsilon_{y y}}{2} \\
\varepsilon_{\|} & =\varepsilon_{z z} \\
\varepsilon_{\text {avg. }} & =\frac{\varepsilon_{\|}+2 \varepsilon_{\perp}}{3}
\end{array}\right\} .
$$

Figs. 4(a)-4(f) show the calculated real and imaginary parts of dielectric functions plotted as a function of incident photon energy for Blal and $14 / \mathrm{mmm}$ phases using GGA and LDA schemes calculated using pseudopotential and fullpotential approaches. We do not observe any significant difference between the spectra calculated using pseudopotential and full-potential approaches. This supports the robustness of our calculation. Calculated spectra for GGA and LDA are also qualitatively identical with certain differences in the intricate details. We, therefore, limit our discussion within the GGA results since the same would be applicable to LDA as well.

For the ferroelectric phase, a comparison between the calculated and experimental dielectric spectra reveals that (i) there is difference in the position of the major features of the spectra and (ii) there is intensity difference as well. While the difference in intensity could arise from a number of factors, such as nature and quality of sample, temperature, difference between experimental and ground state structural parameters, type of approximation scheme used in the firstprinciples calculations, and type of broadening used in the experimental and calculated data, the difference in peak position is chiefly attributed to the underestimation of energy band gap by LDA and GGA techniques, an inherent drawback of such calculations. If the calculated spectra are shifted to higher energy by $1 \mathrm{eV}$, we find good agreement between experimental data and the calculations. Thus, our further analysis would ignore the underestimation of band gap and corresponding mismatch of the peak positions with respect to the experimental spectra and would concentrate more on the origin of the optical spectra. Our calculation on the ferroelectric phase also reveals that there are numerous peaks in the imaginary part of the dielectric function versus energy plot. In order to identify the peaks, we fitted multiple Lorentzian functions and labelled them. The goodness of fitting for all cases is $>0.99$. Table I lists the position of the peaks of the xx component of $\varepsilon^{\prime \prime}$ computed using GGA. Fig. 4(a) shows that $\varepsilon^{\prime \prime}$ spectra for three principal components have peaks at different energy values attributed to different optical transitions allowed at those energies. The anisotropy in the intensity of dielectric spectra, in Fig. 4(a), results in different optical constants along principal crystallographic directions (not shown here). For instance, refractive, indices, extinction coefficients, reflectivity, and real parts of optical 

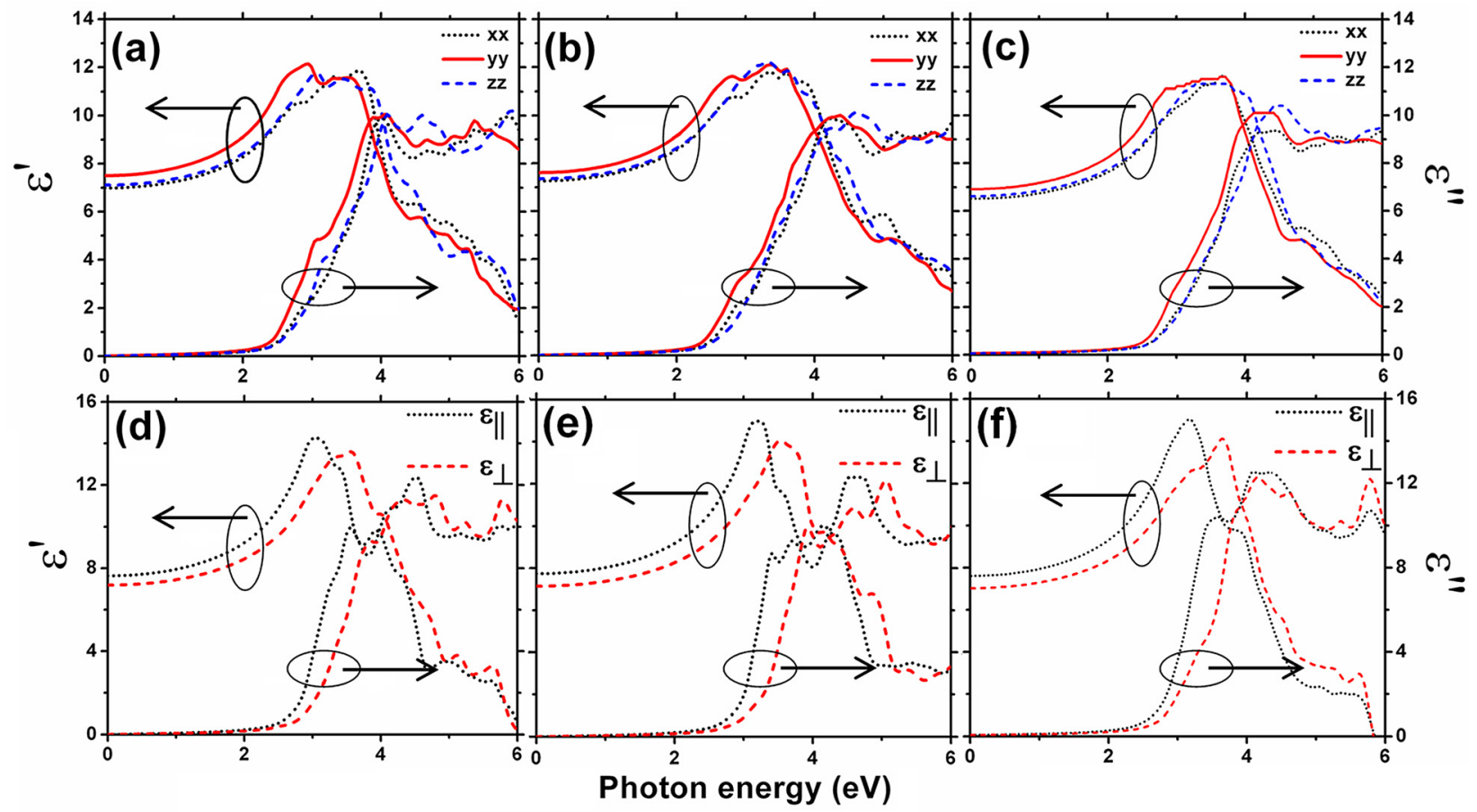

FIG. 4. Real and imaginary parts of dielectric function plotted as a function of incident photon energy for ferroelectric Blal ((a)-(c)) and paraelectric $14 / \mathrm{mmm}$ ((d)-(f)) phases calculated using GGA ((a) and (d)) and LDA ((b) and (e)) methods within vasp and (c) and (f) calculated using GGA within WIEN2k.

conductivities along three principal crystallographic directions demonstrate that while $\mathrm{xx}$ and $\mathrm{zz}$ components of the above optical constants are similar, the magnitudes of yy component differs significantly in Blal symmetry of BiT. This result is consistent with the work of Singh $e t$ al. ${ }^{27}$ where a co-ordinate transformation to Blal symmetry allows the comparison.

In a similar manner, we also calculated dielectric spectra of the paraelectric $14 / \mathrm{mmm}$ phase. Tetragonal symmetry of the system allows only two components of the dielectric spectra, viz., $\varepsilon_{\perp}$ and $\varepsilon_{\|}$as defined in Eq. (5). Fig. 4(d) shows real and imaginary components of dielectric function for tetragonal $14 / \mathrm{mmm}$ symmetry calculated using GGA. Comparison of both real and imaginary components of $\varepsilon_{\perp}$ and $\varepsilon_{\|}$depicts that the high symmetry phase of BiT demonstrates significant anisotropy in the optical properties, which is further substantiated by the anisotropic optical constants (not the shown here). Similar to the ferroelectric phase, we identified the prominent peaks in the imaginary $\varepsilon_{\|}$spectra and listed them in Table I.

TABLE I. Peak positions and peak labeling in $\varepsilon^{\prime \prime}$ spectra of Blal and I4/mmm structures.

\begin{tabular}{llcl}
\hline \hline Blal & $(\mathrm{eV})$ & $I 4 / \mathrm{mmm}$ & $(\mathrm{eV})$ \\
\hline $\mathrm{B}_{1}$ & 2.93 & $\mathrm{I}_{1}$ & 3.21 \\
$\mathrm{~B}_{2}$ & 3.38 & $\mathrm{I}_{2}$ & 3.55 \\
$\mathrm{~B}_{3}$ & 3.97 & $\mathrm{I}_{3}$ & 4.07 \\
$\mathrm{~B}_{4}$ & 4.6 & $\mathrm{I}_{4}$ & 4.51 \\
$\mathrm{~B}_{5}$ & 5.04 & $\mathrm{I}_{5}$ & 5.03 \\
$\mathrm{~B}_{6}$ & 5.41 & $\mathrm{I}_{6}$ & 5.38 \\
$\mathrm{~B}_{7}$ & 5.9 & $\mathrm{I}_{7}$ & 5.83 \\
\hline \hline
\end{tabular}

To identify the origin of these peaks, we subsequently performed electronic structure calculations on the ground state structures of the above two phases. Figs. 5(a) and 5(b) show the parts of the band structures along with the transitions that resulted the above described optical activities in ferroelectric Blal and paraelectric $14 / \mathrm{mmm}$ BiT, respectively. Figs. 5(c) and 5(d) shows corresponding site projected density of states. The calculations were done using GGA.
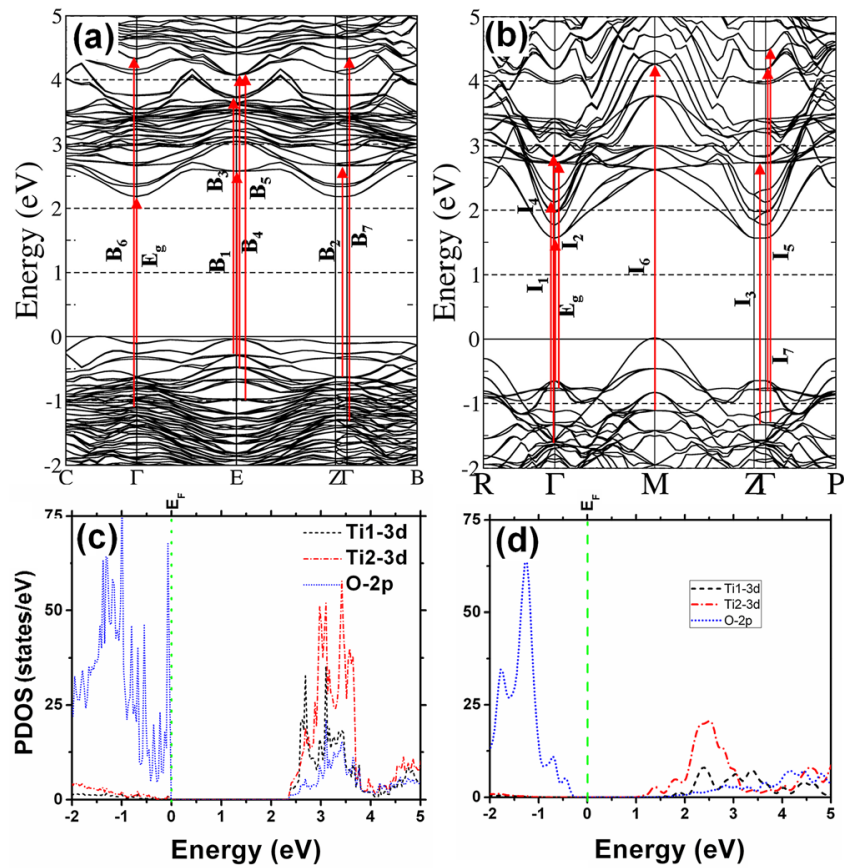

FIG. 5. Partial DOS and band structures of (a) ferroelectric (Blal) and (b) paraelectric $(I 4 / \mathrm{mmm})$ bismuth titanate calculated using GGA. 
Corresponding LDA plots are similar with minute differences and therefore not shown here. Detailed discussions on the density of states and band structures of the two phases can be found elsewhere. ${ }^{24}$ The uppermost part of the VB consists mainly of O $2 p$ states. Above the Fermi level, the $\mathrm{CB}$ is dominated by Ti $3 d$ states where contribution from Ti2 ion is more significant than that of Til. Noticeable amount of Bi $6 p$ and $\mathrm{O} 2 \mathrm{p}$ states are also present here. Therefore, we propose that major optical transition would involve $\mathrm{O} 2 \mathrm{p}, \mathrm{Bi}$ $6 \mathrm{p}$, and Ti $3 \mathrm{~d}$ states.

Crystal structure of BiT consists of alternate perovskite and fluorite layers and the density of $\mathrm{TiO}_{6}$ octahedra is different in crystallographic $c$-direction with respect to the other two directions. Such anisotropic structural feature has been reported to result in anisotropic physical properties such as electrical conductivity. ${ }^{23}$ Although, the density of $\mathrm{TiO}_{6}$ octahedra is identical along $a$ and $b$ directions, the orientation of $\mathrm{TiO}_{6}$ octahedra is different in those two directions. Such orientation difference of Ti-O bonds results in preferential development of spontaneous polarization along crystallographic $a$ direction and not in $b$ direction in BiT. ${ }^{24}$ Further, we notice that, in $14 / \mathrm{mmm}$ structure, optical responses are identical in $a$ and $b$ directions with symmetrical Ti-O bonds. Thus, it is plausible that the orientation differences among the Ti-O bonds lead to different transition behavior of $\mathrm{O} 2 \mathrm{p}$ to $\mathrm{Ti} 3 \mathrm{~d}$, primarily responsible for the optical response of $\mathrm{BiT}$, rendering anisotropic optical behavior in BiT.

\section{CONCLUSIONS}

To summarize, we performed a combined experimentaltheoretical study on the optical properties of ferroelectric and paraelectric phases of bismuth titanate. Our work shows that bismuth titanate demonstrates anisotropic optical properties in both ferroelectric as well as paraelectric phases. The observed optical property could be attributed to the O2p-Ti3d electronic transition while optical anisotropy is attributed to the asymmetrically oriented Ti-O bonds in the unit cell.

\section{ACKNOWLEDGMENTS}

The work was supported by Department of Science and Technology, Govt. of India through project number SR/S2/CMP-0098/2010. Authors thank Surajit Sarkar for his assistance in ellipsometry measurement. S.A. thanks CSIR National Physical Laboratory, New Delhi for financial assistance.

${ }^{1}$ J. F. Scott and C. A. Paz de Araujo, Science 246, 1400-1405 (1989).

${ }^{2}$ B. H. Park, B. S. Kang, S. D. Bu, T. W. Noh, J. Lee, and W. Jo, Nature 401, 682-684 (1999).

${ }^{3}$ C. A. P. de Araujo, J. D. Cuchiaro, L. D. McMillan, M. C. Scott, and J. F. Scott, Nature 374, 627-629 (1995).

${ }^{4}$ A. D. Rae, J. G. Thompson, R. L. Withers, and A. C. Willis, Acta Crystallogr. Sec. B 46, 474-487 (1990).

${ }^{5}$ A. Fouskova and L. E. Cross, J. Appl. Phys. 41, 2834-2838 (1970).

${ }^{6}$ S. Scott, W. A. Schulze, and J. V. Biggers, Ferroelectrics 38, 765-768 (1981).
${ }^{7}$ P. K. Ghosh, A. S. Bhalla, and L. E. Cross, in Dielectric Properties of RF Sputtered Bismuth Titanate Thin Films (1986), pp. 596-598.

${ }^{8}$ V. K. Seth and W. A. Schulze, IEEE Trans. Ultrason., Ferroelectr., Freq. Control 36, 41-49 (1989).

${ }^{9}$ A. Huanosta, O. Alvarez-Fregoso, E. Amano, C. Tabares-Munoz, M. E. Mendoza-Alvarez, and J. G. Mendoza-Alvarez, J. Appl. Phys. 69, 404-408 (1991).

${ }^{10}$ P. S. Nesterenko, S. B. Rastoropov, and A. I. Sokallo, Ferroelectrics 154, 177-181 (1994).

${ }^{11}$ P.-H. Xiang, Y. Kinemuchi, and K. Watari, J. Electroceram. 17, 861-865 (2006).

${ }^{12}$ Q. Y. Tang, Y. G. Li, Y. M. Kan, G. J. Zhang, and P. L. Wang, Wuji Cailiao Xuebao/J. Inorg. Mater. 22, 595-598 (2007).

${ }^{13}$ M. Yamaguchi, T. Nagamoto, and O. Omoto, Thin Solid Films 300, 299-304 (1997).

${ }^{14}$ S. E. Cummins and L. E. Cross, Appl. Phys. Lett. 10, 14-16 (1967).

${ }^{15}$ S. E. Cummins and L. E. Cross, J. Appl. Phys. 39, 2268-2274 (1968).

${ }^{16}$ G. W. Taylor and A. Miller, Proc. IEEE 58, 1220-1229 (1970).

${ }^{17}$ M. Gospodinov, V. Marinova, V. Sainov, and P. Sveshtarov, Mater. Res. Bull. 28, 445-449 (1993).

${ }^{18}$ S. Y. Wu, W. J. Takei, M. H. Francombe, and S. E. Cummins, Ferroelectrics 3, 217-224 (1972).

${ }^{19}$ H. Gu, D. Bao, S. Wang, D. Gao, A. Kuang, and X. Li, Thin Solid Films 283, 81-83 (1996).

${ }^{20}$ W. S. Choi, M. F. Chisholm, D. J. Singh, T. Choi, G. E. Jellison, and H. N. Lee, Nat. Commun. 3, 689 (2012).

${ }^{21}$ S. Y. Yang, L. W. Martin, S. J. Byrnes, T. E. Conry, S. R. Basu, D. Paran, L. Reichertz, J. Ihlefeld, C. Adamo, A. Melville, Y. H. Chu, C. H. Yang, J. L. Musfeldt, D. G. Schlom, J. W. Ager III, and R. Ramesh, Appl. Phys. Lett. 95, 062909-3 (2009).

${ }^{22}$ A. Shrinagar, A. Garg, R. Prasad, and S. Auluck, Acta Crystallogr. Sec. A 64, 368-375 (2008).

${ }^{23}$ Y. Noguchi, M. Soga, M. Takahashi, and M. Miyayama, Jpn. J. Appl. Phys. 44, 6998 (2005).

${ }^{24}$ A. Roy, R. Prasad, S. Auluck, and A. Garg, J. Phys.: Condensed Matter 22, 165902 (2010).

${ }^{25}$ Y. Shen, D. R. Clarke, and P. A. Fuierer, Appl. Phys. Lett. 93, 102907-3 (2008).

${ }^{26}$ M. Takahashi, Y. Noguchi, and M. Miyayama, Jpn. J. Appl. Phys., Part 1 41, 7053 (2002).

${ }^{27}$ D. J. Singh, S. S. A. Seo, and H. N. Lee, Phys. Rev. B 82, 180103 (2010).

${ }^{28}$ S. H. Brewer and S. Franzen, Chem. Phys. 300, 285-293 (2004).

${ }^{29}$ S. Saha, T. P. Sinha, and A. Mookerjee, J. Phys.: Condensed Matter 12, $3325(2000)$.

${ }^{30}$ M.-Q. Cai, Z. Yin, M.-S. Zhang, and Y.-Z. Li, Chem. Phys. Lett. 401, 405-409 (2005).

${ }^{31}$ J. G. E. Jellison and F. A. Modine, Appl. Phys. Lett. 69, 371-373 (1996).

${ }^{32}$ R. O. Jones and O. Gunnarsson, Rev. Mod. Phys. 61, 689 (1989).

${ }^{33}$ G. Kresse and J. Furthmüller, Phys. Rev. B 54, 11169 (1996).

${ }^{34}$ G. Kresse and D. Joubert, Phys. Rev. B 59, 1758 (1999).

${ }^{35}$ P. E. Blöchl, Phys. Rev. B 50, 17953 (1994).

${ }^{36}$ P. Hohenberg and W. Kohn, Phys. Rev. 136, B864 (1964).

${ }^{37}$ W. Kohn and L. J. Sham, Phys. Rev. 140, A1133 (1965).

${ }^{38}$ J. P. Perdew and Y. Wang, Phys. Rev. B 45, 13244 (1992).

${ }^{39}$ J. P. Perdew, K. Burke, and Y. Wang, Phys. Rev. B 54, 16533 (1996).

${ }^{40}$ D. M. Ceperley and B. J. Alder, Phys. Rev. Lett. 45, 566 (1980).

${ }^{41}$ W. H. Press, B. P. Flannery, S. A. Teukolsky, and W. T. Vetterling, Numerical Recipes (Cambridge University Press, New York, 1986).

${ }^{42}$ H. J. Monkhorst and J. D. Pack, Phys. Rev. B 13, 5188 (1976).

${ }^{43}$ C. Jia, Y. Chen, and W. F. Zhang, J. Appl. Phys. 105, 113108-4 (2009).

${ }^{44}$ Y. Zang, D. Xie, Y. Chen, X. Wu, G. Li, and D. Plant, Integr. Ferroelectr. 133, 73-80 (2012).

${ }^{45}$ C. Kittel, Introduction to Solid State Physics (John Wiley \& Sons, Inc., USA, 2005)

${ }^{46}$ S. Bhagavantam, Crystal Symmetry and Physical Properties (Academic Press, London, 1966).

${ }^{47}$ J. F. Nye, Physical Properties of Crystals (Oxford University Press, Oxford, 1985) 